\title{
La base conceptuelle des noms abstraits. Vers la postulation du continuum massif $\leftrightarrow$ comptable
}

\author{
Elmustapha Lemghari \\ Université Cadi Ayyad \\ Faculté Polydisciplinaire de Safi \\ Lemghari.m@hotmail.com
}

\section{Introduction}

L'examen du couple massif/comptable du côté des noms abstraits a donné lieu à deux options différentes. Selon la première option (J. Pelletier (1979); T. Parson (1979); R. Montague (1979), entre autres), l'opposition massif/comptable ne s'applique guère aux noms abstraits. J. Pelletier (1979) fait remarquer à cet égard que la distinction philosophique est vague du point de vue des tests ontologiques qui s'appliquent uniquement aux substances matérielles. La seconde option (J. Moravcsik (1973) ; C.-Y. Chen (1973) ; H. Bunt (1985), etc.) stipule en revanche que la distinction en question convient également aux noms abstraits mais incline à considérer que les abstraits sont tous massifs. Dans cette optique, M. Prandi (1987 : 148 ) souligne que : «si on les envisage sous le profil du comportement des introducteurs (...) les noms désignant des processus physiques (la lumière, la chaleur) et les noms abstraits (la vertu, la sagesse) se comportent comme des noms de masse ».

Notre objectif ici est de montrer conformément aux principes méthodologiques de la Grammaire Cognitive (G. C.) que la distinction massif/comptable s'applique également aux noms abstraits, mais qu'elle gagne davantage à être traitée en termes de continuum qu'en termes d'opposition nette et tranchée. Pour ce faire, trois moments essentiels seront prévus. Pour un souci de clarté, nous rappellerons dans le premier moment, quoique très brièvement, l'apport de la thèse du continuum massif $\leftrightarrow$ comptable dans le domaine des noms concrets. Dans le second, nous examinerons respectivement les noms abstraits massifs prototypiques et les noms comptables prototypiques. L'accent sera mis essentiellement sur leurs conceptions basiques qui autorisent à les ranger dans deux catégories diamétralement opposées. Quant au troisième moment, il sera réservé à une classe de noms qui, de par leurs possibilités combinatoires avec les marqueurs externes de massivité et de comptabilité, fonctionnent comme des intermédiaires, c'est-àdire comme des noms qui vérifient à la fois les propriétés du massif et du comptable. L'intérêt portera dans cette perspective tant sur le statut ontologique des noms abstraits que sur leurs occurrences discursives.

\section{Mise au point}

Il est utile, pour la clarté de l'exposé, de procéder à une mise au point en matière de la problématique de la distinction massif/comptable qui permettra, chemin faisant, d'expliciter la position que nous avons adoptée à ce sujet dans des travaux antérieurs. L'idée défendue stipule que la distinction massif/comptable, vu la structure sémantique des noms et la complexité des problèmes qu'elle soulève, peut être beaucoup mieux abordée sous l'angle de la scalarité et du continuum que sous celui de la radicalité des oppositions franches. Ce choix nous semble garanti par l'existence en français d'une classe de noms intermédiaires, noms qui sont difficilement classifiables exclusivement comme massifs ou comme comptables mais plutôt comme noms à la fois massifs et comptables, c'est-à-dire comme noms qui vérifient similairement les critères ontologiques et sémantico-référentiels qui définissent les deux types de noms ${ }^{1}$.

La littérature est très abondante sur le sujet, mais l'originalité de l'idée défendue réside, contrairement à toutes les présentations classiques, dans cette nouvelle perspective où la problématique est revue et traitée. Pour aller vite, nous rappellerons brièvement les résultats auxquels nous avons abouti et qui 
peuvent se résumer au point suivant : en français, une bonne description des noms comptables et massifs doit prendre en ligne de compte non seulement les aspects canoniques des noms mais aussi leurs structures internes qui sont souvent complexes. C'est bien cet oubli, pense-t-on, qui a décidé de l'évolution de toutes les recherches, qui se sont consacrées à cette problématique, vers la division de la catégorie nominale en deux sous-catégories adverses. Tout bien considéré, les noms en français présentent trois structures différentes, une structure homogène qui caractérise les noms massifs, une structure hétérogène qui caractérise les noms comptables et, finalement, une structure intermédiaire qui définit la troisième catégorie et qui se prête à la fois comme homogène et hétérogène. Cette dernière est ainsi appelée parce qu'elle comporte des noms qui vérifient indifféremment la batterie des critères, conçus pour tracer des frontières infranchissables entre les deux catégories en question ${ }^{2}$.

Il s'agit, comme on le voit, de relancer le vieux débat de la distinction massif/comptable sur la base de cette nouvelle thèse et de réexaminer ainsi tous les problèmes qui se révèlent réellement rétifs à une opposition binaire massif/comptable (la recatégorisation, la polysémie, la généricité, entre autres faits de la langue). Aux risques de redites, on rappellera en d'autres mots que le postulat de base consiste à distinguer en français trois classes de noms, la classe nominale massive prototypique, la classe nominale comptable prototypique et la classe nominale intermédiaire. Une telle idée autorise à décrire la structure interne des noms dans toute leur complexité ; par conséquent, tant la nature scalaire ${ }^{3}$ des noms massifs prototypiques que la nature ambivalente des noms intermédiaires sont impliquées et méritent, du coup, d'être évoquées. Renoncer au traitement des comptables et des massifs en termes d'opposition rigide a pour avantage en effet d'échapper à la menace de circularité à laquelle semble conduire infailliblement toute analyse qui ne rompt pas avec la tradition aristotélicienne - phylogenèse oblige, dirait-on - en faveur d'oppositions binaires souvent irrévocablement établies.

Dans le domaine des noms massifs et comptables concrets, à titre de rappel, le continuum massif nous semble assuré par des noms du type de linge et papier, qui s'accommodent indifféremment et des marqueurs externes de massivité et des marqueurs externes de comptabilité, comme il ressort des exemples suivants ${ }^{4}$ :

1. a. Larsonneau était devenu un viveur élégant avec du linge éblouissant. (Zola, la Curée)

b. D'ailleurs, le grand enchanteur Altorino ne prit-il pas pour une procession de fantômes des linges qu'une blanchisseuse de Biscaye avait mis à sécher au clair de lune. (Hugo, Amy Robsart)

2. a. On aperçut un vieillard en habit noir. D'une main il tenait un rameau vert, de l'autre un papier et les secouait avec agitation. (Flaubert, l'Education sentimentale)

b. Il arrivait encore parfois qu'elle lui écrivit d'un restaurant ou d'un hôtel sur du papier qui en portait le nom imprimé. (Proust, Un amour de Swan)

Le bien-fondé de l'opposition massif/comptable en termes de la conception aristotélicienne se trouve aisément remis en question par les exemples (1-a-b) et (2-a-b). En effet, la batterie des tests ontologiques ${ }^{5}$ et sémantico-référentiels invoqués pour décrire cette opposition se révèlent insuffisants pour rendre compte de l'apparition de ces noms tantôt sous l'aspect massif, tantôt sous l'aspect comptable, comme en témoigne leur distribution indifféremment avec les marqueurs externes de l'une et de l'autre classe. Deux raisons, qui jouent sur deux plans distincts, permettent, si on y regarde de très près, de conclure à la souscatégorisation ambivalente de ces noms: le premier plan est celui du $\mathrm{N}$ où opèrent les critères ontologiques et le second, celui du $\mathrm{SN}$ où les noms massifs ou comptables de départ peuvent acquérir le caractère inverse et être ainsi versés discursivement dans la catégorie opposée.

Vérifions! $\mathrm{Au}$ niveau du $\mathrm{N}$, paradoxalement, l'application des deux principes ontologiques fondamentaux, en l'occurrence la référence cumulative et la division en sous-ensemble, aux noms linge et papier se révèlent inefficaces quant à distinguer le comptable du massif : en effet, l'addition de $d u$ linge à $d u$ linge donne toujours $d u$ linge; le nom linge se prête alors comme fondamentalement massif. Inversement, tout porte à conclure qu'après l'addition de deux ou de plusieurs pièces de linge, le résultat 
est des linges, référent individué, et non du linge, référent non-individué. Sous cet angle, linge est un nom intrinsèquement comptable.

Au niveau du SN, la réification catégorielle (la recatégorisation) ${ }^{6}$, n'en sort pas plus victorieuse que les tests ontologiques : de même qu'on ne peut trancher, sur pièces (c'est-à-dire sur la base d'un mode de recatégorisation bien défini), que les SN des linges et un papier dans (1-b) et (2-a) sont dérivés respectivement des SN $d u$ linge et $d u$ papier, en vertu d'un type donné de recatégorisation comptable $\rightarrow$ massif, de même il est impossible de conclure à une recatégorisation inverse de du linge et du papier à partir des emplois comptables des linges et un papier. S'il arrive pourtant qu'on conteste cet argument, il va falloir trouver à chacun d'eux une sous-catégorisation propre soit en tant que massif, soit en tant que comptable et d'où seront dérivés par recatégorisation les emplois inverses.

Il s'ensuit que de tels exemples conduisent à abandonner, comme trop radicale, l'opposition traditionnelle massif/comptable, d'inspiration aristotélicienne, au profit de la perspective du continuum massif $\leftrightarrow$ comptable et d'envisager, afin d'en vérifier le bien-fondé, la possibilité de l'élargir à d'autres secteurs, comme celui des noms abstraits, objet du présent travail.

\section{Les noms abstraits massifs et comptables prototypiques}

Dans le cadre de la G.C, les noms abstraits peuvent, au même titre que les noms concrets, avoir des emplois grammaticaux différents, en ce sens qu'ils sont susceptibles de co-occurrer comme comptables ou massifs selon qu'ils désignent une région délimitée ou une région ${ }^{7}$ non délimitée dans l'espace $d^{\prime}$ instanciation ${ }^{8}$. Eu égard à la nature ontologique et aux comportements morphosyntaxiques de ces noms, on distingue, d'une part, les noms massifs prototypiques et, d'autre part, les noms comptables prototypiques.

\subsection{Les noms abstraits massifs prototypiques}

\subsubsection{Les noms d'activités}

Les noms abstraits massifs prototypiques désignent généralement des procès dont la structure interne se présente comme étant homogène. Ce sont principalement les noms d'activités du type de marche, natation...

3. a. La marche est encore le meilleur des exercices. (Gide, Le P. Robert)

b. Elle en avait pour une demi-heure de marche. (Green, ibid.)

Le statut massif du nom marche est assuré par l'homogénéité de la région profilée et l'absence de frontières délimitatrices : les états constitutifs de l'activité exprimée par le procès marche sont conçus comme étant continus. D'où la possibilité d'étendre ou de contracter la région profilée sans modifier pour autant la caractérisation basique du procès désigné. Sous cet angle, la région profilée par un nom abstrait massif du type de marche est conçue comme désignant une substance abstraite, en ce sens qu'elle est similaire aux substances physiques dénotées par des noms concrets massifs. C'est pour cette raison que les noms d'activités sont dits noms de grandeurs extensives, conçus comme ayant une continuité dans le temps (cf. D. Van de Velde, 1996). En effet, de même que beaucoup de sable occupe plus d'espace que peu de sable, de même beaucoup de marche occupe plus de temps que peu de marche.

Certes une substance physique se manifeste de manière discontinue dans son domaine d'instanciation : ses différentes instanciations forment ensemble une région non pas en termes de la contiguïté spatiale de ses entités interconnectées mais en vertu de leur uniformité qualitative, c'est-à-dire leur location commune dans l'espace de qualité ${ }^{10}$. Etant donné que les noms abstraits du type de marche dénotent des procès, leur domaine d'instanciation est le temps : la substance abstraite qu'ils désignent est, semblable 
en cela aux substances concrètes, distribuée de façon discontinue dans le domaine d'instanciation. Or, la catégorie (i.e. l'activité ou le procès dénotés) est instanciée chaque fois qu'on s'engage dans le procès en question. Autrement dit, malgré leurs intervalles temporels, ces instanciations infinies constituent une région abstraite grâce à leur uniformité qualitative.

En somme, les instanciations d'une substance physique sont d'ordre matériel ; le domaine évoqué pour leur caractérisation qualitative se rapporte à des propriétés comme la couleur, la viscosité, la transparence, la rigidité, etc. Par contre, la substance abstraite désignée par un nom comme marche, par exemple, ne dispose d'aucune existence matérielle; elle se manifeste seulement à travers l'occurrence d'un procès. Ses entités constitutives ne sont par conséquent pas des locations matérielles mais plutôt des relations, et plus précisément, les états du procès profilé. Il s'ensuit que la caractérisation qualitative de la substance abstraite réside dans la conception du procès désigné : ce n'est qu'à travers leurs états internes représentatifs qu'une série de relations peuvent être reconnues comme instanciant la substance concernée.

L'extension dans le temps qui caractérise les noms d'activités se laisse confirmer par leur association au quantificateur beaucoup de :

\section{J'ai fait beaucoup de marche dans ma vie. (D. Van de Velde, 1996)}

L'expression faire beaucoup de marche signifie consacrer beaucoup de temps à faire de la marche où beaucoup de temps résulte de la sommation de nombreuses périodes de temps séparées par des intervalles. Les intervalles susceptibles de séparer les périodes de temps constituent autant d'instanciations de la substance dénotée par le procès désigné. Ces différentes périodes forment ensemble une région abstraite non délimitée en termes de leur uniformité qualitative. Du coup, le nom marche est fondamentalement massif. Ce trait peut, par ailleurs, être corroboré par la possibilité de verser le nom, en vertu du processus du trieur universel ${ }^{11}$, dans la catégorie comptable, comme dans Une marche pénible, longue, etc.

\subsubsection{Les noms de couleurs}

Si les noms de couleurs sont comptés parmi les membres de la classe massive prototypique, c'est pour autant qu'ils en partagent les principaux traits, à savoir qu'ils résistent à la pluralisation et s'accommodent naturellement de l'article partitif $d u$, deux critères unanimement retenus par les linguistes en matière de spécification des deux natures, massive et comptable :

5. * les blancs, les jaunes...de ce tableau.

6. M. de Ferrare et toute sa suite avait du jaune et du rouge. M.de Guise parut avec de l'incarnat et du blanc (...) M.de Nemours avait du jaune et du noir. (Mme de la Fayette, la Princesse de Clèves)

Eu égard à leurs caractères de noms basiquement massifs, les noms de couleurs profilent des régions non délimitées dans l'espace d'instanciation. Comme les couleurs sont souvent dites être dans les choses colorées ${ }^{12}$, celles-ci leur servent d'espace d'instanciation. Dans (7)

\section{Il y' a du blanc dans ce tableau,}

la région que profile le nom blanc est conçue comme étant non délimitée dans l'espace d'instanciation représenté par la scène visuelle que constitue la configuration tableau. La sensation de couleur en question est par conséquent susceptible, à la manière d'une substance physique massive, d'être étendue ou contractée sans que pour autant la conception que reçoit le nom en question soit modifiée.

\subsection{Les noms abstraits comptables prototypiques}

\subsubsection{Les noms désignant des procès épisodiques}


Le caractère comptable des noms épisodiques procède directement de leur caractérisation basique; un nom épisodique profile une région comprenant les états constitutifs d'un procès perfectif, et du moment que de tels procès sont intrinsèquement délimités, la région qu'ils profilent est elle-même délimitée. En d'autres mots, le procès épisodique présuppose des limites inhérentes à l'ensemble des entités constitutives.

Les noms épisodiques sont généralement les noms d'accomplissements. Ces noms dénotent également des procès, c'est-à-dire qu'ils sont des activités dans la mesure où ils disposent d'une étendue temporelle. La différence entre les noms d'activités et les noms d'accomplissements tient par conséquent à leurs statuts de noms massifs et de noms comptables respectivement : les noms d'activités sont conçus comme non délimités dans l'espace temporel tandis que les noms d'accomplissements sont perçus comme ayant une limite propre dans l'espace temporel; d'où leur conception basique de noms intrinsèquement comptables. Considérons à titre d'illustration l'énoncé suivant :

8. Absorbé dans ma douce rêverie, je prolongeais fort avant dans la nuit ma promenade sans m'apercevoir que j'étais las. (Rousseau, les Promenades solitaires).

Le nom promenade est un nom d'accomplissement qui désigne un procès épisodique, procès qui comporte dans sa signification un terme naturel, une fin. En conséquence, il est intrinsèquement comptable, puisque la région qu'il profile se prête comme étant nécessairement délimitée dans l'espace temporel. En somme, le sémantisme des noms d'accomplissements implique l'idée d'une fin inhérente qui doit nécessairement être atteinte pour que les procès dénotés puissent être dits accomplis.

Par ailleurs, eu égard aux limites inhérentes qui caractérisent les régions profilées par les noms d'accomplissements, ceux-ci se révèlent, sur la plan discursif, incompatibles avec les compléments temporels ayant la structure sémantique de ceux qu'introduit la préposition pendant suivie d'un nom de mesure temporelle qui exige l'homogénéité du nom spécifié. Soient les énoncés (9) et (10) :

9. * Je veux faire un aller et retour pendant trois heures.

10. Je veux faire de la marche pendant trois heures. ( D. Van de Velde, 1996: 223)

L'inadéquation de l'énoncé (9) participe de l'impossibilité de combiner le spécifieur de mesure temporelle pendant trois heures, qui exige que les états constitutifs du procès désigné par le nom soient homogènes, avec les noms aller et retour, noms d'accomplissements dont les états constitutifs sont caractérisés par des limites propres intrinsèques à leurs conceptions basiques. Ce spécifieur implique en effet, soit la répétition, soit la poursuite ininterrompue d'une même chose à chaque moment d'une durée indéterminée. A ce propos, Van de Velde (1996: 222) fait remarquer que : « s'il fallait construire un sens pour l'expression faire un aller et retour pendant trois heures, il faudrait diviser la durée de trois heures en un nombre indéterminé de périodes au long desquelles se répète identiquement ou se poursuit, sans solution de continuité, l'action de faire un aller et retour, ce qui serait contradictoire dans le premier cas avec l'usage du singulier, dans le second avec la signification même de l'expression ». En revanche, dans (10), le spécifieur temporel pendant trois heures est bien compatible avec le nom marche. Ceci s'explique par le fait que le nom marche désigne un procès dont les états constitutifs sont conçus comme étant homogènes, c'est-à-dire que la région profilée est exempte de limites propres qui puissent la délimiter dans l'espace temporel.

Inversement, les spécifieurs de mesure temporelle en en, qui exigent généralement que le nom qu'ils spécifient soit constitué d'états hétérogènes, apparaissent, en fait, bien appropriés avec les noms d'accomplissements :

11. Je veux faire un aller et retour en trois heures.

12. * Je peux faire de la marche en trois heures. (Van de Velde, 1996: 223)

La signification des spécifieurs de mesure temporelle, compatibles avec les noms comptables, est en parfaite harmonie avec celle des noms d'accomplissements, étant donné que le rôle des spécifieurs 
comptables est précisément de faire coïncider les limites d'une durée avec les limites d'une action déterminée.

\subsubsection{Les noms désignant des faits}

En français, certains noms abstraits ne s'accommodent pas du partitif $d u$, preuve qu'ils n'appartiennent pas à la catégorie des noms susceptibles de dénoter des grandeurs extensives ou intensives (infra. Section 4). Ces noms qui désignent des propriétés non susceptibles d'accroissement ou de diminution, c'est-à-dire les noms qui désignent des régions qui ne satisfont pas aux propriétés de replicabilité et de contractibilité, acquièrent une interprétation qui est régulièrement et nécessairement celle d'un fait ou d'un contenu propositionnel. Tel est le cas des noms du type de mort, rondeur, immortalité, etc.

13. a. * Il y avait déjà de la mort en lui. (Van de Velde 1996: 131)

b. Je ne vous lasserais pas mourir, j'ai de la vie pour deux et je vous infuserais mon sang s'il fallait. (Balsac, la Maison du Chat qui pelote).

14. La finitude d'un monde resserré entre le macrocosme et le microcosme. (Foucault, le P. Robert)

15. Je contemple d'en haut le globe en sa rondeur. (Baudelaire, le P. Robert).

16. Par la suite, Prométhée acquit l'immortalité, que lui céda le centaure Chiron. (Dictionnaire de la mythologie grecque et romaine)

Dans (13-a-b), quoi que les noms vie et mort soient des noms d'états, seul le nom vie est susceptible de prendre l'article partitif $d u$. Le nom vie doit cet emploi à la grandeur intensive qu'il dénote, c'est-à-dire aux degrés d'accroissement ou de diminution spécifiant sa structure interne. Les noms finitude, rondeur, immortalité, etc., par contre ne s'associent pas au partitif $d u$, attendu qu'ils ne sont ni les noms de grandeurs intensives, dans la mesure où ils ne sont pas susceptibles d'accroissement et de diminution, ni les noms de grandeurs extensives, puisqu'ils n'ont aucune temporalité propre. Ce sont simplement et uniquement des noms de faits. Leur incompatibilité avec le partitif $d u$ les range du côté des noms fondamentalement comptables.

\section{Les noms intermédiaires}

La thèse du continuum massif $\leftrightarrow$ comptable se laisse confirmer du côté des noms abstraits, par l'existence de deux types de noms qui sont indifféremment basiquement massifs et comptables en dehors de toute forme de réification discursive : les noms de qualités et les noms d'états ${ }^{13}$, noms dits de grandeurs intensives (cf.Van de Velde, 1996). Leur sous-catégorisation discursive en massif ou en comptable dépend en effet essentiellement de leurs bases conceptuelles ou, plus précisément, du domaine cognitif où ils sont susceptibles d'être instanciés. On note à ce sujet que la base conceptuelle d'un prédicat en G.C. est composée d'un faisceau de domaines cognitifs. Ces domaines sont autant de structures sémantiques présupposées dans une base conceptuelle donnée et qui représentent le contexte informatif du prédicat concerné. Il s'ensuit que s'il existe des noms abstraits intermédiaires, c'est bien en vertu de leurs bases conceptuelles qui sont caractérisées par au moins deux domaines cognitifs fondamentaux, un domaine où ils sont massifs et un autre où ils sont comptables.

\subsection{Statut massif des noms de grandeurs intensives}

Les noms concrets qui se combinent naturellement avec le partitif $d u$ sont certes des noms basiquement massifs ; ils forment une catégorie homogène. Ce sont principalement des noms de matières qui désignent des grandeurs continues, matériellement extensibles dans l'espace physique. $D u$ sable, de l'eau, par exemple, se présentent comme occupant réellement des locations dans l'espace d'instanciation.

Eu égard à leurs étendues dans l'espace d'instanciation, les régions que profilent les expressions $d u$ sable, de l'eau répondent aux propriétés de contractibilité et de replicabilité sans qu'aucun changement n'affecte leurs structures internes. Il en est de même des noms abstraits qui dénotent des grandeurs 
intensives. Ces noms sont ainsi appelés parce qu'ils sont susceptibles d'accroissement et de diminution sans occuper pour autant plus au moins d'espace physique. Autrement dit, le propre d'une grandeur intensive est d'être capable d'augmentation et de réduction sans extension corrélative ni dans l'espace physique ni dans le l'espace temporel ${ }^{14}$.

Parallèlement aux noms concrets dont l'usage du partitif parvient à désigner une quantité indéterminée de la substance concernée, les noms abstraits de qualités et d'états, en vertu des grandeurs intensives qu'ils dénotent, répondent, lorsqu'ils sont associés au partitif $d u$, à la visée d'un degré indéterminé de la qualité ou de l'état en question :

17. Vous m'avez donné de la passion (...) elle dure encore, je n'ai jamais pu vous donner de l'amour. (Mme de la Fayette, la Princesse de Clèves)

18. Il vernit lui-même ses bottes (...), se substituant à Frédéric (...) par une singulière évolution intellectuelle où il y avait à la fois de la vengeance, de la sympathie, de l'imitation et de l'audace. (Flaubert, l'Education sentimentale)

Les noms de qualités et d'états associés au partitif $d u$ dans (17) et (18) sont conçus comme ayant des degrés indéterminés sur l'échelle d'intensité. Cette intensité procède de l'étendue homogène et non délimitée qui caractérise les régions profilées. Sous cet angle, les noms abstraits de grandeurs intensives sont fondamentalement massifs. A preuve, les mêmes déterminants qui s'associent aux noms basiquement massifs de matières pour indiquer une quantité indéterminée (i.e. le partitif $d u$ ) ou des quantités relativement déterminées (i.e. les quantificateurs indiquant des quantités relatives : un peu de, beaucoup de, etc.) sont compatibles avec les noms abstraits de grandeurs intensives, autrement dit les noms massifs concrets et les noms abstraits intensifs ont le même comportement morphosyntaxique, comme il ressort de (19) et (18).

19. a. Ses relations (...) pourraient lui rendre un peu de prix aux yeux d'Odette. (Proust, Un Amour de Swan)

b. Elle rapportait de la rue un peu du vacarme et de l'animation du boulevard. (Zola, la Curée)

20. a. Le jeune homme avait beaucoup de mémoire. (Flaubert, Mme Bovary)

b. Nous le vîmes qui travaillait en conscience, cherchant tous les mots dans le dictionnaire et se donnant beaucoup de mal. (Flaubert, ibid.)

\subsection{Statuts comptables des noms de grandeurs intensives}

\subsubsection{Interprétations des noms de qualités en actes}

L'occurrence des noms de qualités en tant que noms comptables trouve sa validation dans le mode de profilation de la région désignée, qui se prête sous cet emploi comme région basiquement délimitée. Ceci suppose par conséquent, qu'un même nom puisse être différemment interprétable selon qu'il est basiquement massif ou comptable.

L'interprétation typique selon laquelle le nom de qualité apparaît sous le giron du comptable est l'interprétation en acte. Considérons à titre d'exemple les énoncés suivants :

21. Aigri par de longues injustices, souffrant de vielles blessures, et toujours regrettant l'Empereur, il dégorgeait sur son entourage les colères qui l'étouffaient. (Flaubert, l'Education sentimentale)

22. Les atrocités qui se commettent dans la prison de Boda. (Gide, le P. Robert)

23. On crut qu'il payait la totalité de ses dettes, qu'il faisait des folies pour elle. (Zola, la Curée).

Les noms injustices, atrocités et folies utilisés au pluriel dans (21), (22) et (23) sont interprétés en actes. La pluralisation ici correspond naturellement à ce qu'implique la possibilité d'utiliser le singulier un, comme il apparait dans les phrases suivantes : 
24. Une injustice demeure collée à toute souffrance même la plus méritée aux yeux des hommes. (Camus, l'Homme révolté)

25. Et le partage de ces reliques, où il retrouvait confusément les formes de ses formes, lui semblait une atrocité. (Flaubert, l'Education sentimentale)

26. Il dit que se marier actuellement serait une folie. (Flaubert, Ibid.)

L'emploi de un avec les noms justice, atrocité et folie impose nécessairement des limites sur les grandeurs intensives qu'ils dénotent. Ces limites tirent leur origine en effet de l'occurrence des noms concernés non en tant que noms de qualité mais plus précisément en tant qu'actes qualifiés. Il s'agit, tout bien considéré, d'actes dont l'aspect comptable est dû à leur statut d'unités discrètes ayant en ellesmêmes le principe de leur limite, ce qui, d'une part, les rapproche des noms épisodiques et, d'autre part, justifie, pris sous la parité du comportement morphosyntaxique, leur similarité avec les comptables concrets ${ }^{15}$. Aussi, à la différence de leurs emplois massifs correspondants, sont-ils rétifs à la propriété d'accroissement et de réduction. D'où leur structure de noms intrinsèquement comptables.

On en conclut que les noms de qualités du type de injustice, atrocité et folie sont basiquement comptables lorsqu'ils s'appliquent à désigner des actes qualifiés. Etant ainsi interprétés, ils cessent d'être des grandeurs intensives et dénotent, en contrepartie, des individus abstraits, discontinus et dénombrables qui profilent conséquemment des régions conçues comme intrinsèquement délimitées dans l'espace temporel.

\subsubsection{Occurrences comptables des noms d'états}

Les noms d'états (généralement les noms de sentiments) sont également des noms intermédiaires, pour autant qu'ils co-occurrent tantôt comme comptables, tantôt comme massifs. Rappelons ici que la possibilité des noms abstraits intermédiaires de bénéficier d'emplois grammaticaux différents (i.e. à la fois massifs et comptables) tient précisément au mode de profilation de la région désignée ; celle-ci peut être conçue soit comme délimitée, soit comme non délimitée dans quelques domaines cognitifs.

Généralement, les noms d'états en occurrences massives, désignent des sensations émotionnelles; les régions qu'ils profilent sont non délimitées dans l'espace émotif. Considérons à titre d'illustration l'énoncé suivant :

27. Vous ne sauriez douter (...) que j'ai de la joie de vous voir. (Mme de la Fayette, la Princesse de Clèves)

Dans (27), le nom joie apparaît comme massif. Ceci s'explique par le fait que la substance dénotée est perçue comme étant non délimitée dans l'espace émotif qui est son domaine primordial sous cet emploi. Aussi la substance en question est-elle instanciée chaque fois que le locuteur expérimente la sensation décrite. Les fragments d'une telle expérience sont considérés en G.C. comme étant les entités massives qui composent la substance concernée.

Il faut remarquer toutefois que les noms d'états qui sont des grandeurs intensives susceptibles d'augmentation et de réduction sans extension corrélative ni dans l'espace temporel ni dans l'espace physique entretiennent malgré tout un rapport privilégié avec le temps. C'est effectivement ce rapport qui se trouve à l'origine de l'instauration de leur statut de noms fondamentalement comptables, lequel statut se manifeste par la possibilité qu'ils ont de s'associer à l'opérateur de discontinuité un sans qu'aucune adjonction d'ordre qualitatif ne soit nécessaire. Soient les énoncés suivants :

28. a. (...) afin de couvrir sa lâcheté, il étalait une colère où peu à peu il se trouvait pris lui-même. (Flaubert, l'Education sentimentale)

b. Frédéric eut un remords, et se montra assidu au journal. (Flaubert, ibid.)

c. Il eut un attendrissement en voyant le premier fiacre. (Flaubert, ibid.) 


\section{d. Puis ils étaient vaincus d'avance par la perte du zaimph; ceux mêmes qui ne s'en souciaient avaient senti une angoisse et comme un affaiblissement. (Flaubert, Salammbô)}

Dans (28-a-d), les noms colère, remords, attendrissement, angoisse et affaiblissement s'appliquent à désigner une fraction du temps occupé par les états qu'ils dénotent. Une fraction de temps comporte naturellement en elle-même le principe de sa limite ; les noms précités qui l'expriment sont appréhendés comme les noms d'individus. D'où leur caractère commun de noms comptables.

Contentons-nous, pour plus de détails, d'analyser à titre d'exemple, l'énoncé (28-c). Le statut comptable du nom attendrissement est assuré par la nature de la région profilée : dans (28-c), en raison de la fraction close du temps occupé par l'état exprimé par le nom attendrissement, la région profilée est perçue comme étant délimitée dans l'espace temporel. Dans le cas contraire, c'est-à-dire, lorsque le nom attendrissement apparaît avec le partitif $d u$, comme dans (29) :

\section{9. (...) et gagné par l'émotion de la vielle fille, il arrivait à sentir pour lui de l'attendrissement.} (Flaubert, l'Education sentimentale),

la région profilée se présente effectivement comme non délimitée dans l'espace émotif. La distribution du partitif $d u$ avec le nom attendrissement correspond à un degré indéterminé de la grandeur intensive que le nom dénote.

On l'aura constaté, sous l'aspect massif, les noms d'état et de sentiment sont conçus comme susceptibles d'augmentation et de diminution dans l'espace émotif qui est derechef leur domaine primordial dans ce cas, comme l'est d'ailleurs l'espace physique pour les substances concrètes. Mais, sous l'aspect comptable, ils se présentent comme instanciés dans un autre espace, à savoir l'espace temporel. La variation de l'espace d'instanciation, du coup, décide de leur statut massif ou comptable. On n'assiste point ici à une conversion discursive massive $\rightarrow$ comptable, étant donné que le trait comptable et le trait massif de ces noms sont le colloraire de leur statut intermédiaire ; autrement dit, ils leur sont inhérents.

Une précision s'impose pourtant au sujet des noms intensifs et de leur statut intermédiaire : Tous les noms de grandeurs intensives ne sont pas obligatoirement des intermédiaires, les intermédiaires étant les seuls noms qui, malgré leur caractère intensif, entretiennent des rapports privilégiés avec le temps, c'est-à-dire, les noms qui sont capables de profiler des régions délimitées dans l'espace temporel. Si colère, dépression, paralysie, etc., se combinent aisément avec l'opérateur de discontinuité un sous l'interprétation quantitative, il n'en va pas de même pour perplexité, frustration, etc., ce qui prouve que seuls les premiers profilent des régions délimitées dans l'espace temporel et accèdent, de ce fait, au statut des intermédiaires.

Il s'ensuit qu'entre les deux pôles prototypiques respectivement massif et comptable, il y a lieu de réserver une place charnière à des niveaux intermédiaires représentés par une classe de noms dont la structure est indifféremment basiquement massive et comptable. L'existence d'une telle classe de noms concourt par conséquent à traiter la problématique du couple massif/comptable en termes de continuum au lieu d'opposition rigide.

\section{Conclusion}

Nous nous sommes proposé dans cet article de tenter de corroborer la thèse du continuum massif $\leftrightarrow$ comptable du côté des noms abstraits. Trois classes de noms abstraits ont été relevées, la classe du massif prototypique, celle du comptable prototypique et la classe des noms intermédiaires.

(i)- La classe du massif prototypique est formée, d'une part, par les noms qui désignent des activités (i.e. les noms de grandeurs extensives qui profilent des régions non délimitées dans l'espace temporel) et, 
d'autre part, par les noms de couleurs qui paraissent partager les principaux traits du massif prototypique. Ces noms profilent des régions dans l'espace d'instanciation représenté par les scènes visuelles (i.e. les entités colorées).

(ii)- La classe du comptable prototypique est constituée par les noms d'accomplissements, c'est-à-dire, par les noms qui désignent des procès épisodiques. Eu égard aux limites intrinsèques aux conceptions des procès épisodiques, ceux-ci profilent des régions perçues comme étant délimitées dans l'espace temporel. La classe du comptable prototypique est également formée par un ensemble de noms qui ne s'accommodent jamais du partitif $d u$. Ces noms sont uniquement et simplement des noms de faits ou de contenu propositionnel.

(iii)- Enfin, la classe des intermédiaires comporte les noms qui sont à la fois massifs et comptables, présidant ainsi à l'établissement du continuum massif $\leftrightarrow$ comptable. La classe des intermédiaires se compose de deux types de noms : ceux qui désignent des qualités et ceux qui désignent des états et des sentiments. Leurs occurrences massives ou comptables reposent essentiellement sur la variation des domaines cognitifs qui constituent le contexte informatif de leurs bases conceptuelles, domaines cognitifs primordiaux en effet où la région dénotée se prête soit comme délimitée, soit comme non délimitée.

\section{Références bibliographiques}

Beauseroy, D. (2008). Les noms abstraits intensifs ou "noms statifs": (non) projection du nombre et interprétation, in Durand J. Habert B., Laks B. (éds), Actes du CMLF 2008, 2389-2406.

Bunt, H. (1985). Mass terms and Model theoretic semantics, Cambridge : Cambridge University Press.

Borillo, A. (1989). Notions de «massifs » et «comptables » dans la mesure temporelle. In J. David et G. Kleiber (éds), Termes massifs et termes comptables, Klinksieck, Paris, 215-238.

Cheng C.-Y. (1973). Comments of Moravcsik's paper. In J. Hintikka et al. (éds) Approaches to natural language, Dordrecht-Boston-Reidel.

David J. et Kleiber, G. (éds.) (1989). Termes massifs et termes comptables, Paris : Klincksieck.

Flaux, N. et Van de Velde, D. (2000). Les noms en français : esquisse de classement, Paris : Ophrys.

Galmiche, M. (1986). Note sur les noms de masse et le partitif, Langue française, 72, 40-53.

Galmiche, M. (1987). A propos de la distinction massif/comptable, Modèles linguistiques, 9, 2, 179-203.

Galmiche, M. (1989). Massif/comptable : de l'un à l'autre et inversement. In J. David et G. Kleiber (éds.), Termes massifs et termes comptables, Paris : Klincksieck, 63-77.

Kleiber, G. (1990a). La sémantique du prototype, Paris : PUF.

Kleiber,G. (1990b). L'article le générique. La généricité sur le mode massif, Genève : Droz.

Kleiber, G. (1999). Problèmes de Sémantique. La polysémie en questions, Paris: Presses Universitaires du Septentrion.

Kleiber, G., 2008, Petit essai pour montrer que la polysémie n'est pas un sens interdit. In Durand J. Habert B., Laks B. (éds), Actes du CMLF 2008, 87-100.

Langacker, R.W. (1987). Foundations of Cognitive Grammar. Vol 1, Standford University Press.

Langacker, R.W. (1991). Foundations of Cognitive Grammar ; Vol. 2. Descriptive Application, Standford University Press.

Lemghari Elm. (2001). Les noms massifs et les noms comptables: approche sémantique et cognitive, Thèse de Doctorat, Rabat.

Lemghari Elm. (sous presse ). Le français, une langue à classificateurs. La distinction massif/comptable en question. 
Lemghari M. (sous presse). La complexité sous-catégorielle des noms massifs et comptables. Quelques faits de polysémies lexicales régulières.

Montague, R. (1979). The Proper Treatment of Mass Terms in English. In F. Pelletier (éds), Mass Terms : Some Philosophical Problems, Dordrecht : Reidel.

Moravcsik, J. (1973). Mass Terms in English. In J. Hintikka et al. (éds.), Appproaches to Natural language, Dordrecht : Reidel.

Parsons, T. (1979). An analysis of Mass Terms and Amount Terms. In F.J. Pelletier (éd.), Mass Terms : Some Philosophical Problems, Dordrecht : Reidel, 137-166.

Pelletier, F.J. (1975). Non-Singular reference : Some Preminaries, Philosophia, 5 , 4, 451-465.

Pelletier, F.J. (éd.) (1979). Mass Terms : Some Philosophical Problems. Dordrecht : Reidel.

Prandi, M. (1987). Sémantique du contresens, Paris : Editions de Minuit.

Quine, W. V. (1960). Word and object, Cambridge : MIT Press.

Wilmet, M. (1989), Le problème des noms abstraits. In J. David et G. Kleiber (éds.), Termes massifs et termes comptables, Paris : Klincksieck.

${ }^{1}$ Force est de constater que la thèse des noms intermédiaires rejoint dans une large mesure l'idée que G. Kleiber (1999 : 112) semble sinon aborder du moins effleurer lorsqu'il souligne que : " La distinction massif / comptable est à traiter à deux niveaux distincts [...]. Au niveau lexical tout d'abord, où chaque $\mathrm{N}$ se voit intrinsèquement marqué soit comme comptable (cf. chaise), soit comme massif (cf. sable), soit encore comme comptable et massif (cf. pain) ».

${ }^{2}$ Il s'agit en effet tant des critères ontologiques que des critères syntaxico-sémantiques (Voir, pour plus de détails, J. David et G. Kleiber : 1989).

${ }^{3}$ La catégorie massive prototypique est caractérisée par une structure scalaire qui est due aux conceptions basiques des noms. Pour peu qu'on considère la suite suivante air > eau > poussière > quincaillerie $>$ bétail, on n'est plus fondé de recourir au critère ontologique de l'homogénéité pour les sous-catégoriser tous ensemble dans la catégorie massive, dans la mesure où leurs structures référentielles sont progressivement individuées les unes par rapport aux autres. Cette scalarité contribue à distinguer au sein de la classe massive prototypique des sous-catégories plus au moins homogènes s'organisant en une sorte de hiérarchie qui va du plus massif au moins massif, en l'occurrence : les volatiles (air) $\rightarrow$ les liquides (eau) $\rightarrow$ les solides (sable).

${ }^{4}$ Le lecteur aura remarqué que les documents dépouillés sont très variés. Cette hétérogénéité du corpus répond, pour nous, à un impératif d'authenticité, d'autant plus que le recours à des exemples forgés, pour appuyer telle ou telle idée, ne peut être exempt d'un certain artifice de linguiste. A cette raison s'ajoute le fait que, abstraction faite de l'époque à laquelle appartient l'auteur cité, les emplois qui nous concernent ici ne sont pas tombées en désuétude et se rencontrent souvent sous des plumes contemporaines

${ }^{5}$ La thèse ontologique s'insère dans le cadre de la tendance logico-philosophique qui sous-catégorise un nom donné comme massif et /ou comptable en vertu de l'examen de son statut ontologique, et ce, par l'application de certains principes dont particulièrement : le principe de référence cumulative, le principe de référence distributive, le principe de référence homogène, et les principes de qualification homogène et de qualification indéfinie. Tous ces principes ontologiques se réduisent aux propriétés fondamentales de la divisibilité homogène et de la divisibilité hétérogène, largement connues et discutées depuis Aristote.

${ }^{6}$ Voir, pour plus de détails du phénomène de recatégorisation, M. Galmiche (1989).

${ }^{7}$ En G.C. la définition schématique proposée pour le nom est qu'il désigne (profile) une région dans quelque domaine; celle-ci est définie abstraitement comme un ensemble d'entités interconnectées, susceptibles d'être instanciées dans un domaine cognitif donné.

${ }^{8}$ Le domaine d'instanciation est le domaine où les instances d'un type sont conçues comme ayant une location qui les distingue d'autres instances. Autrement dit, c'est le domaine cognitif primordial où un nom donné profile une région comme délimitée, c'est-dire massive ou non délimitée, c'est-à-dire comptable. 
${ }^{9}$ Dans le cadre de la G.C, les noms massifs et les noms comptables s'opposent de par quatre propriétés sémanticoréférentielles, la replicabilité, la contractibilité, l'homogénéité et la délimitation. Ces propriétés, qui peuvent se ramener, en dernière analyse, aux principes de référence cumulative et distributive, sont également ontologiques, à cette différence près qu'elles sont systématisées, c'est-à-dire intégrées à la grammaire.

${ }^{10}$ L'espace quantitatif est un espace multidimensionnel conçu en termes de paramètres qualitatifs, c'est-à-dire qu'il désigne un ensemble de propriétés qu'une entité peut posséder (i.e. solidité, couleur, viscosité, etc.).

${ }^{11}$ Le trieur universel est proposé, sur le modèle du broyeur universel de J. Pelletier (1975), par H. Bunt (1985). Ce processus a pour effet de rendre compte du transfert du massif au comptable en opérant des distinctions à l'intérieur d'une substance donnée pour aboutir à diverses variétés. Les distinctions opérées sont, par conséquent, d'ordre qualitatif, comme dans Un vin blanc où le numératif un opère un découpage à l'intérieur de la catégorie dénotée, découpage qui s'applique à référer à une sous-espèce qualitativement distincte. (Voir, pour une critique de ce précédé, M. Galmiche : 1989).

${ }^{12}$ Le fait même que les noms de couleurs existent dans les choses colorées conduit certains chercheurs à les considérer comme moins abstraits que les noms purement abstraits. Van de Velde (1996:149) fait remarquer à cet égard que : « du côté du concret les noms de matières tendent vers l'abstraction, et du côté de l'abstrait les noms de couleurs tendent vers la concrétisation ».

${ }^{13}$ Les différences qui distinguent les qualités des états sont moins probantes. Généralement, une qualité apparaît comme plus stable et plus permanente qu'un état. Par exemple, la patience d'un individu est plus stable et plus durable que sa tristesse. Pareillement, la dureté d'une pierre est plus longue que sa chaleur. Notons de plus que le passage des qualités aux états et vis-versa est fréquent: le nom saleté, à titre d'exemple, désigne une qualité dans Etre d'une saleté repoussante et un état dans Etre dans une saleté repoussante.

${ }^{14}$ N. Flaux et D. Van de Velde (2000) argumentent en faveur de l'absence d'étendue temporelle pour les noms intensifs, à la différence des noms extensifs, par l'impossibilité de les combiner avec l'adverbe beaucoup sous une lecture durative ou fréquentative, comme il ressort de la comparaison de Paul a fait beaucoup de marche (= Paul a marché longtemps/souvent) avec Paul aime beaucoup son chien (=*Paul aime son chien longtemps/souvent).

15 Voir, pour le comportement morphosyntaxique des noms intensifs en occurrence massive et en occurrence comptable, l'article de D. Beauseroy (2008). 